\title{
Aspects Epidemiologiques Des Traumatismes Lies Aux Accidents De La Voie Publique Chez Les Adultes Au Centre Hospitalier Universitaire De Reference Nationale De N'Djamena (Chu-Rn), Tchad
}

Moussa Kalli
Service de Chirurgie générale, CHU de Référence Nationale, N’Djaména

$$
\text { (CHU-RN) }
$$

\section{Andjeffa Valentin}

Service d'Orthopédie et Traumatologie au CHU-RN, N’Djaména (CHU-RN)

\section{Seid Younous}

Service de Chirurgie générale, CHU de Référence Nationale, N’Djaména

$$
\text { (CHU-RN) }
$$

\section{Adjougoulta Bonté}

Service d’Anesthésie et de Réanimation, au CHU-RN, N’Djaména (CHU-

$\mathrm{RN})$

\section{Bruno Mantou}

Kambel Djibdouna

Sadié Ismael Guire

Yasmine Abdoulaye

Abdelaziz Wiché

Service de Chirurgie générale, CHU de Référence Nationale, N’Djaména

$$
\text { (CHU-RN) }
$$

\section{Choua Ouchemi}

Service de Chirurgie générale, Centre Hospitalier Universitaire de Référence

Nationale, N’Djaména (CHU-RN),

Faculté de sciences de la Santé Humaine de Ndjamena (FSSH)

Doi:10.19044/esj.2021.v17n25p396

Submitted: 22 June 2021

Accepted: 10 July 2021

Published: 31 July 2021
Copyright 2021 Author(s)

Under Creative Commons BY-NC-ND 4.0 OPEN ACCESS

Cite As:

Kalli M., Valentin A., Younous S., Bonté A., Mantou B., Djibdouna K., Guire S.I., Abdoulaye Y., Wiché A. \& Ouchemi C.(2021). Aspects Epidemiologiques Des Traumatismes Lies Aux Accidents De La Voie Publique Chez Les Adultes Au Centre Hospitalier Universitaire De Reference Nationale De N'djamena (Chu-Rn), Tchad. European Scientific Journal, ESJ, 17(25), 396. 


\section{Résumé}

Introduction: les accidents de trafic routier constituent un problème de santé publique.

Objectif: décrire le profil épidémiologique, les circonstances de survenues et les facteurs associés aux accidents de la route dans la ville de N’Djaména.

Méthodes: il s’agissait d'une étude transversale et descriptive ayant inclus tous les accidentés de la voie publique admis au service des urgences chirurgicales du centre hospitalier universitaire de référence nationale de N’Djaména (CHU-RN) durant une période de six mois. Les paramètres sociodémographiques, cliniques et thérapeutiques étaient décrits et confrontés à la littérature.

Résultats: en tout, 5772 patients étaient admis aux urgences chirurgicales dont 2575 étaient victimes d'AVP soit une fréquence de 44,6 \%. L’âge moyen était de 28,5 ans avec des extrêmes de 16 et 75 ans. Le sexe masculin représentait $77 \%$ et le sex ratio était de 3,4. Une moto était impliquée chez 70,3\% des cas. Les motocyclistes étaient les victimes les plus concernées (76\%). Les principaux facteurs associés aux accidents de la voie publique étaient l'excès de vitesse et la transgression du code de la route dans respectivement $23 \%$ et $21 \%$ des cas. La moitié des patients étaient emmenés aux urgences à bord d'un véhicule personnel. Le transport en ambulance concernait $4 \%$ des cas.

Les lésions de moindre gravité (écorchures, plaies superficielles) étaient les plus nombreuses (52\%). Les lésions principales étaient surtout localisées au crane (21\%). Le casque n’était pas utilisé par les victimes. La mortalité hospitalière était de 4,1\%.

Conclusion: les accidents de la circulation demeurent un véritable problème de santé publique même en période de restriction sanitaire. L'utilisation de casques et de couloirs cyclistes devrait contribuer à la réduction des accidents et de leur gravité, particulièrement à la baisse des traumatismes crâniens.

Mots clés: Accident De La Voie Publique, Polytraumatisme, Traumatisme Crânien, Covid-19, Tchad 


\section{Epidemiologic Aspects Of Injuries Related To Road Traffic Accidents Among Adults At The University Hospital Of National Reference Of N'djamena (Chu-Rn), Chad}

\section{Moussa Kalli}

Service de Chirurgie générale, CHU de Référence Nationale, N’Djaména

$$
\text { (CHU-RN) }
$$

\section{Andjeffa Valentin}

Service d'Orthopédie et Traumatologie au CHU-RN, N’Djaména (CHU-RN)

\section{Seid Younous}

Service de Chirurgie générale, CHU de Référence Nationale, N’Djaména

$$
\text { (CHU-RN) }
$$

\section{Adjougoulta Bonté}

Service d'Anesthésie et de Réanimation, au CHU-RN, N’Djaména (CHU-

$\mathrm{RN}$ )

Bruno Mantou

Kambel Djibdouna

Sadié Ismael Guire

Yasmine Abdoulaye

Abdelaziz Wiché

Service de Chirurgie générale, CHU de Référence Nationale, N’Djaména (CHU-RN)

\section{Choua Ouchemi}

Service de Chirurgie générale, Centre Hospitalier Universitaire de Référence Nationale, N’Djaména (CHU-RN)

Faculté de sciences de la Santé Humaine de Ndjamena (FSSH)

\section{Abstract}

Introduction: Road traffic accidents are a public health problem.

Objective: To describe the epidemiological profile, circumstances of occurrence and factors associated with road traffic accidents in the city of N'Djamena during COVID-19 pandemic.

Methods: This was a cross-sectional and descriptive study that included all road traffic accidents admitted to the surgical emergency department of the national reference university hospital of N'Djaména (CHU$\mathrm{RN}$ ) during a six-month period. Sociodemographic, clinic and therapeutic aspects were described and compared to the literature.

Results: A total of 5,772 patients were admitted to the surgical emergency department, of whom 2,575 were victims of MVA, i.e. a frequency of $44.6 \%$. The mean age was 28.5 years with extremes of 16 and 75 years. Males accounted for $77 \%$ and the sex ratio was 3.4. A motorbike was involved 
in $70.3 \%$ of the cases. Motorcyclists were the most affected victims (76\%). The main factors associated with the road accidents were speeding and traffic violations in $23 \%$ and $21 \%$ of cases respectively. Half of the patients were taken to the emergency room in a private vehicle. Transport by ambulance concerned $4 \%$ of cases.

Less serious injuries (abrasions, superficial wounds) were the most numerous (52\%). The main injuries were mainly located in the skull (21\%). Helmets were not used by the victims. Hospital mortality was $4.1 \%$.

Conclusion: Traffic accidents remain a real public health problem. The use of helmets and bicycle lanes should contribute to the reduction of accidents and their severity, particularly to the reduction of head injuries.

Key words: Road Traffic Accident, Polytrauma, Head Trauma, Covid-19, Chad

\section{Introduction}

L'urbanisation rapide et anarchique des grandes villes africaines et le non-respect du code de la route par des usagers sont souvent à l'origine de traumatismes parfois graves dus aux accidents de la voie publique (AVP). Les AVP sont des évènements causant au moins une victime, survenant sur une voie ouverte à la circulation publique et impliquant au moins un véhicule (ODIMBA, 2007 ; SCURFIELD, 2004). Ils ont toujours fait l'objet d'une grande préoccupation de la part des autorités médicales et publiques. Un rapport récent de l'Organisation Mondiale de la Santé (OMS) décrit les traumatismes de la voie publique comme une épidémie gravissime, car ils sont responsables dans le monde de 1,2 million de décès par an. Ils représentent la première cause de décès dans le monde chez des personnes âgées de 5 à 24 ans (SCURFIELD, 2004). Au Tchad, particulièrement à N’Djaména qui est la ville la plus peuplée avec plus d'un million d'habitants, on constate que le nombre de traumatismes par AVP commence à prendre des proportions inquiétantes. Ainsi, les accidents de la circulation en 2008 étaient estimés à $67,5 \%$ de toutes les admissions chirurgicales et les motocyclistes ont été les plus concernés avec 42,9\% des cas (KABORO, 2008). En 2014, vue la fréquence de traumatismes crâniens par AVP en milieu hospitalier un plaidoyer a été fait au près des autorités publiques pour le port obligatoire de casque chez les usagers de motocyclettes (CHOUA, 2014). Compte tenu de ce constat, il nous a paru nécessaire d'étudier les traumatismes par AVP dans la ville de N’Djaména pour proposer des mesures de prévention en vue de réduire leur fréquence. 


\section{Patients Et Methode}

Il s'agissait d'une étude descriptive transversale sur une période de six (6) mois, de juillet à décembre 2020 menée au service des urgences du CHURN de N’Djaména. Etaient inclus les patients des deux sexes âgés de plus de 15 ans reçus pour AVP, consentants et présentant un traumatisme corporel. Les patients admis aux urgences pour des pathologies autres que le traumatisme par AVP, les patients non consentants et les patients n'ayant pas présenté de blessures après examen clinique n'ont pas été inclus dans l'étude. Les variables étudiées étaient: l'âge, le sexe, la profession, la provenance, le mode de recrutement, les circonstances de survenues de l'accident (lieu, moment, véhicule ayant provoqué le traumatisme, port ou non de casque et ceinture de sécurité), les facteurs associés à l'AVP et les données cliniques (délai de la prise en charge, topographie et nature des lésions) et la mortalité. Les données étaient recueillies à partir des dossiers médicaux et des registres des urgences chirurgicales. Les données étaient collectées et saisies à l'aide du logiciel Excel et office 2016.

Un consentement écrit était obtenu chez tous les patients adultes, ou auprès des tuteurs pour les patients âgés de moins de 18 ans. Si la victime d'AVP était un adulte inconscient, le consentement était obtenu auprès du parent accompagnateur. Les données étaient traitées et utilisées dans un but d'analyse scientifique dans le strict anonymat.

\section{Resultats}

\section{Caractéristiques sociodémographiques}

Au cours de cette étude: 5772 patients étaient admis aux urgences chirurgicales dont 2575 victimes d'AVP, soit une fréquence de 44,6\%. Une moyenne de 14 blessés par AVP chaque jour a été observée. La tranche d'âge la plus représentée était celle de 21 à 30 ans. L’âge moyen était de 28,5 ans avec des extrêmes de 16 et 75 ans. Les deux sexes sont atteints, avec une prédominance masculine (77\%) soit un sex-ratio de 3,4. Parmi les victimes, 2482 (96,4\%) provenaient de la ville de N’Djaména. 1417 (55\%) AVP étaient survenus entre $16 \mathrm{~h}$ et $18 \mathrm{~h}$. La majorité des accidents étaient enregistrés au mois de décembre et juillet respectivement chez $611(24 \%)$ et $496(19,2 \%)$ des cas. Le groupe élèves et étudiants était le plus représenté (44,6\%), suivis des chauffeurs de mototaxis avec 500 (19,4\%) patients. Les motocyclistes étaient majoritairement impliqués dans les AVP (76\%) comme reporté au tableau I.

Tableau I. les engins impliqués dans les accidents de la voie publique

\begin{tabular}{|c|c|c|}
\hline Engins en cause & $\mathbf{N}$ & $\mathbf{\%}$ \\
\hline Moto & 1810 & $70,324,1$ \\
Auto & 620 & \\
\hline Auto-moto & 145 & 5,6 \\
\hline Total & $\mathbf{2 5 7 5}$ & $\mathbf{1 0 0}$ \\
\hline
\end{tabular}


Une moto était impliquée dans la survenue de l’AVP chez 76\% des cas.

Les facteurs associés aux AVP étaient : l'excès de vitesse, la transgression du code de la route et le déplacement pendant la nuit dans respectivement $23,4 \%, 21,2 \%$ et $11 \%$ des cas. La prise de stupéfiants au volant était retrouvée chez 70 victimes soit 3\%. L'état d'ivresse était retrouvé chez 60 patients $(2,3 \%)$ et huit accidentés $(0,3 \%)$ faisaient usage du téléphone au volant. Certains patients avaient plusieurs facteurs à la fois et chez certains aucun facteur n’a été retrouvé (39\%) (tableau II). La ceinture de sécurité était retrouvée chez sept (7) victimes soit $0,3 \%$. Aucun usager de motocyclette accidenté ne portait un casque. Mille deux cents quatre-vingt-sept (50\%) victimes étaient transportées du lieu de l'accident au service des urgences par un véhicule de particuliers (tableau III).

Tableau II. répartition des patients selon les facteurs associés aux AVP.

\begin{tabular}{|c|c|c|}
\hline Facteurs & $\mathbf{n}$ & $\mathbf{\%}$ \\
\hline Indéterminé & 1000 & 39 \\
Excès de vitesse & 607 & $23,4 \%$ \\
\hline Transgression du code de la route & 550 & $21,2 \%$ \\
\hline Déplacement de nuit & 280 & $11 \%$ \\
\hline Prise de drogue & 70 & $3 \%$ \\
\hline Etat d'ivresse & 60 & $2,4 \%$ \\
\hline Utilisation du téléphone au volant & 8 & $0,3 \%$ \\
\hline Total & $\mathbf{2 5 7 5}$ & $\mathbf{1 0 0} \%$ \\
\hline
\end{tabular}

Tableau III. distribution des patients selon les moyens de transport aux urgences

\begin{tabular}{|c|c|c|}
\hline Mode de transport & $\mathbf{N}$ & $\mathbf{\%}$ \\
\hline Véhicule personnel & 1287 & $50 \%$ \\
Taxi & 952 & $37 \%$ \\
\hline Moto & 232 & $9 \%$ \\
\hline Ambulance & 104 & $4 \%$ \\
\hline Total & $\mathbf{2 5 7 5}$ & $\mathbf{1 0 0} \%$ \\
\hline
\end{tabular}

\section{Caractéristiques cliniques, thérapeutiques et pronostics}

La plupart des patients présentait un bon état général (97\%) et un bon état hémodynamique $(98,5 \%)$ à l'admission. Les lésions de moindre gravité (écorchures, plaies superficielles, contusions musculaires) étaient les plus nombreuses (52\%). Le traumatisme crânien isolé, le polytraumatisme et les fractures des membres étaient retrouvés chez respectivement $21 \%, 18 \%$ et 7,5\%. (Tableau IV). Ces cas étaient traités en collaboration avec les anesthésistes réanimateurs et les traumatologues.

Tableau IV. répartition des traumatisés en fonction de la topographie des lésions

\begin{tabular}{|c|c|c|}
\hline Type & $\mathbf{N}$ & $\mathbf{\%}$ \\
\hline $\begin{array}{c}\text { Traumatisme légers(écorchures, contusions } \\
\text { musculaires) }\end{array}$ & 1339 & $52 \%$ \\
\hline Traumatisme crânien isolé & 540 & $21 \%$ \\
\hline
\end{tabular}




\begin{tabular}{|c|c|c|}
\hline Polytraumatisme & 335 & $18 \%$ \\
\hline Fracture de membres & 195 & $7,5 \%$ \\
\hline Traumatisme abdominal et du bassin & 45 & $4 \%$ \\
\hline Traumatisme thoracique & 100 & $3,8 \%$ \\
\hline Traumatisme cervical & 21 & $0,8 \%$ \\
\hline Total & $\mathbf{2 5 7 5}$ & $\mathbf{1 0 0 \%}$ \\
\hline
\end{tabular}

Les patients étaient pris en charge avant la sixième heure dans $96 \%$ des cas. Ils quittaient l'hôpital après les premiers soins dans 55 \% des cas ; 23 \% étaient mis en observation pendant 24-48 heures et $22 \%$ étaient orientés vers les pavillons d'hospitalisation.

Le geste le plus réalisé aux urgences était un pansement avec suture chez $63 \%$ des cas suivi des attouchements chez $37 \%$ des patients, qui recevaient tous du sérum antitétanique. Une immobilisation provisoire au niveau des membres était réalisée chez 195 (7,5\%) des patients au niveau des urgences chirurgicales avant d'être transférés au service de traumatologie ou libérés.

Le taux de mortalité était de 4,1 \% ( $\mathrm{n}=105)$. Les patients décédés étaient tous des motocyclistes sans casque, polytraumatisés avec comme lésion principale un traumatisme crânien grave.

\section{Discussion}

Dans notre pratique, les accidents de la voie publique sont très fréquents, constituant presque la moitié $(44,6 \%)$ des admissions aux urgences chirurgicales. Ce taux élevé d'accident de la circulation s'expliquerait d'abord par l'accroissement du parc automobile urbain et l'augmentation du nombre des engins motorisés à deux roueset au fait que notre centre hospitalier est orienté vers la prise en charge des urgences traumatiques. Plusieurs auteurs dont Mc Greevy et al à Yaoundé (MC GREEVY, 2014), Almeimoune et al (ALMEIMOUNE, 2017) à Bamako, et Tekpa et al. (TEKPA, 2017) à Bangui rapportent des données similaires aux nôtres. Ce taux est inférieur à ceux relevés dans des études antérieures dans le même hôpital du CHU-RN, par Kaboro et al (KABORO, 2008) qui a trouvé 67,9\% et Choua et al (CHOUA, 2014) qui rapportaient $61,2 \%$. L'explication retrouvée est que la présente étude s'est déroulée pendant la pandémie de covid-19 où les AVP ont diminué à cause des mesures restrictives instaurées par le gouvernement. La fermeture des bars, restaurants et le couvre-feu auraient diminué le nombre d'individus circulants dans les rues et par conséquent les occasions d'AVP.

L'âge moyen des patients est de 28,5 ans avec des extrêmes de 16 et 75 ans. Cette prédominance des sujets jeunes dans les AVP est retrouvée par plusieurs auteurs en Afrique subsaharienne (ODIMBA, 2007 ; Mc GREEVY, 2014 ; ALMEIMOUNE, 2017 ; TEKPA, 2017). 
La tendance juvénile masculine des victimes de ce travail a été rapportée aussi par des auteurs au Tchad, en RCA et au Mali (KABORO, 2008 ; TEKPA, 2017 ; MADOUGOU, 2016).

Cette fréquence élevée des accidents chez les hommes s'expliquerait par la différence des effectifs entre les chauffeurs de sexe masculin et ceux de sexe féminin, mais aussi par le comportement à risque des hommes au volant et dans la conduite des engins à deux roues. Les hommes effectuent des multiples déplacements imposés par leurs activités économiques, éducatives ou administratives. Le chômage des diplômés de tous les secteurs peut pousser cette tranche d'âge à s'improviser chauffeurs rémunérés de transport en commun sans aucune notion du code de la route.

La plupart des victimes des AVP a été reçue au service des urgences chirurgicales dans la journée avec un afflux des malades entre $16 \mathrm{~h}$ et $18 \mathrm{~h}$ (55\%). Selon Madougou et al (MADOUGOU, 2016), les accidents étaient plus fréquents après $18 \mathrm{~h}$ dans $37,3 \%$ des cas. La présente étude est réalisée pendant une période de couvre-feu, instauré à partir de $18 \mathrm{~h}$ à cause de la pandémie de Covid-19. La tranche d'heures de $16 \mathrm{~h}$ à $18 \mathrm{~h}$ correspond au moment où la circulation est dense, du fait du retour des travailleurs à la maison généralement fatigués donc moins vigilants et qui se déplacent pour la plupart avec des engins à deux roues.

Par ailleurs, en dehors des grands axes routiers, les rues sont mal ou non éclairées à N’Djamena. Dans cette série le mois de décembre a enregistré le plus d'accidents de la voie publique avec une fréquence de $24 \%$. Ce mois correspond à la période des grandes festivités de fin d'année, ce qui créerait d'avantage d'occasions de circulation routière et de victimes. Cette tendance est retrouvée dans d'autres séries africaines (ODIMBA, 2007 ; Mc GREEVY, 2014 ; ALMEIMOUNE, 2017).

La classe socioprofessionnelle la plus touchée dans ce travail est celle des élèves et étudiants. Cette tendance est rapportée par Almeimoune et al à Bamako, par contre Madougou et al à Yaoundé rapportent plus de non scolarisés (ALMEIMOUNE, 2017 ; MADOUGOU, 2016). Dans la présente série les AVP consécutifs aux motocyclettes représentaient 70\% des accidents. Ce résultat s'expliquerait par l'utilisation fréquente des engins à deux roues par les jeunes à N'Djaména. Les motos ont un coût accessible, les élèves et étudiants les utilisent pour leurs besoins de mobilité, mais aussi pour travailler comme des 'clandoman' ou chauffeurs de taxi-motos comme activité génératrice de revenus.

Des constatations similaires sont rapportées dans la littérature africaine (ODIMBA, 2007 ; KABORO, 2008 ; CHOUA, 2014 ; ALMEIMOUNE, 2017 ; TEPKA, 2017 ; MADOUGOU, 2016). Les usagers de la route qui reportent le plus de lésions post accidentelles sont dans trois quarts des cas (76\%) des motocyclistes. Ces derniers sont vulnérables pour des multiples 
raisons dont le manque d'enveloppe de protection sur la moto, contrairement au véhicule. Ainsi, au cours d'un accident, le motocycliste absorbe toute l'énergie produite par l'impact avec la route et les autres véhicules. Les deux roues constituent l'essentiel des moyens de déplacement à N'Djaména pour la majorité de la population (KABORO, 2004 ; CHOUA, 2014). La conduite de véhicules à deux roues motorisés comporte un risque plus élevé d'accident mortel de la circulation que tout autre mode de transport courant.

On estime que, pour 100 millions d'heures de déplacement, 440 décès de conducteurs de deux-roues motorisés se produisent, contre 75 et 25 décès respectivement pour les cyclistes et les automobilistes (SAUTER, 2005).

Toutes les différentes causes ou facteurs de risque connus d'accidents de trafic routier sont de grande importance. Dans cette étude, pour 39\% des accidents la cause n'est pas établie. L'excès de vitesse est le facteur le plus associé aux AVP (23\%). Il est suivi de la transgression du code de la route et le déplacement la nuit dans respectivement $21,4 \%$ et $11 \%$ des cas. Ce constat a aussi été fait par d'autres auteurs qui ont trouvé que l'excès de vitesse était parmi les causes majeures d'accidents de trafic routier (ODIMBA, 2007 ; TEPKA, 2017 ; MADOUGOU, 2016 ; ILUNGA, 2014). La conduite sous l'emprise de l'alcool ou de toute substance psycho active augmente le risque d'accidents graves ou mortels. L'ivresse au volant n'était retrouvée que chez 2,3\% des cas de cette série. Le dosage d'alcoolémie ne se fait pas systématiquement au CHU-RN. Cependant, en République démocratique du Congo, l'alcool était la cause la plus impliquée dans la survenue des accidents du trafic routier (ILUNGA, 2014 ; MOISE, 2018). Le faible taux d'alcoolisme au volant de cette série par rapport à d'autres études réalisées au Tchad serait lié à notre échantillon et à la période d'étude (KABORO, 2008 ; CHOUA, 2014). En effet, le présent travail s'est déroulé en pleine pandémie de covid19 qui comporte à cause du couvre-feu, une fermeture nocturne des débits de boissons.

Il a été relevé dans cette série huit cas $(0,3 \%)$ d'accidents de la voie publique chez des victimes qui conversaient au téléphone portable pendant la conduite. L'utilisation du téléphone au volant était le facteur le plus incriminé dans la survenue des accidents de trafic routier dans une étude réalisée en Ethiopie (HASSEN, 2011). Il semble que ce phénomène soit récurrent à N'Djaména, les victimes ne le renseigneraient pas pendant l'interrogatoire.

Certains auteurs incriminent l'inexpérience des conducteurs dans la survenue des AVP (KABORO, 2008 ; ALMEIMOUNE 2017). Dans cette série l'expérience des conducteurs n'a pas été évaluée.

Tous les motocyclistes accidentés de la présente série ne portaient pas de casque. Ce comportement reste inchangé depuis une décennie à N’Djamena (KABORO, 2008 ; CHOUA, 2014). Le port de casque diminue la mortalité et les lésions graves après un accident (SAUTER, 2005). Le taux de mortalité 
réel par AVP pourrait être plus élevé, au vu du nombre de patients transportés à l'hôpital en ambulance qui est de seulement $4 \%$. Seuls les cas de décès survenus à l'hôpital ont été pris en considération. Au vu du mode de transport, les traumatisés les plus graves seraient décédés sur les lieux du sinistre. Nous retrouvons par ailleurs qu'après les lésions contusives de moindre gravité, le crâne était la région la plus atteinte suivie des membres inférieurs. Certains auteurs rapportent des résultats similaires (ALMEIMOUNE, 2017; MADOUGOU, 2016). D'autres retrouvent par contre que les lésions prédominantes sont les fractures du membre pelvien (SAUTER, 2005).

Ce travail préliminaire devrait être poursuivi en tenant compte des données pré hospitalières afin d'être fixés sur le taux réel de décès par AVP. Il nous permet cependant de souligner l'effet protecteur du casque chez les motocyclistes, principales victimes des traumatismes crâniens lors des AVP à N’Djaména.

\section{Conclusion}

Les accidents de la voie publique restent un problème majeur de santé publique au Tchad par leur fréquence, malgré leur réduction relative à cause des mesures de restriction en période de Covid-19. Ils sont l'apanage du sujet jeune de sexe masculin. Une motocyclette est souvent impliquée. En raison du taux de mortalité et des conséquences dramatiques sur le plan sanitaire et socio-économique (nombre de journées de travail perdus, augmentation des dépenses de santé publique...), la prévention passe par la sécurisation de la circulation routière. Les autorités devraient veiller à l'application au renforcement de la législation nationale sur les facteurs de risque d'accident (vitesse, conduite en état d'ébriété, port du casque, attache de la ceinture de sécurité) afin d'en réduire la fréquence.

\section{References:}

1. Almeimoune A, Manga MI, Diop Th M, et al. (2017). Aspects épidémiologiques, cliniques des traumatismes liés aux accidents de la circulation routière (ACR) impliquant les motos à Bamako. Rev Afr Anesthésiol Med Urgence; 22(1) :64-67.

2. Choua O, Zounvournaï A, Sidi SK et al. (2014). Accidents de motos à N'Djaména: plaidoyer pour le port de casque. Ann Univ Abd Moum; Série A (25):26-33.

3. Hassen A, Godesso A, Abebe L, Girma E (2011). Risky driving behaviors for road traffic accident among drivers in Mekele city, Northern Ethiopia. BMC Research Notes; 4:535. Doi:10.1186/17560500-4-535. 
4. Ilunga KS, Matungul CP, Mukanya PK et al. (2014). Facteurs associés aux accidents de la route dans la ville de Lubumbashi. Santé publique ;26(6): 889-895.

5. Kaboro M, Dionadji M, Djibrine D, et al. (2008). Bilan de 29 jours de prise en charge de traumatisés par accident de la voie publique. Méd d’Afr Noire ;55(6): 325-331.

6. Madougou S, Chigblo PS ,Tchomtchoua AS et al. (2016). Incidence et impacts des accidents de la voie publique chez les conducteurs de taxi-moto en milieu tropical. Revue de chirurgie orthopédique et traumatologique ;102: 211-4

7. McGreevy J, Stevens KA, Monono ME, et al. (2014). Road traffic injuries in Yaoundé, Cameroon: a hospital-based pilot surveillance study. Injury ;45:1687-92.

8. Moise MV, Kalongo IM, Kambale KJ et al. (2018). Prise en charge des accidentes du trafic routier (ATR) en ville de Butembo, EST de la République démocratique du Congo. Revue Médicale des Grands Lacs; 9(2): 1-6.

9. Odimba EBK (2007). Aspects particuliers des traumatismes dans les pays peu nantis d'Afrique: un vécu chirurgical de vingt ans. E-mém Acad Nat Chirurgie;6(2):44-56.

10. Sauter C, Zhu S, Allen S, Hargarten S, Layde PM (2005). Increased risk of death or disability in unhelmeted Wisconsin motorcyclists. Wis Med J;10492:39-44.

11. Scurfield R, Sleet D (2004). Rapport mondial sur la prévention des traumatismes dus aux accidents de la circulation. Organisation Mondiale de la Santé (OMS). Genève.

12. Tekpa BJD, Diemer HC, Issa Mapouka PA et al. (2017). La mortalité au cours de la circulation routière à Bangui, République Centrafricaine. Méd et Sté Trop; 27:426-430. 